\title{
A Survey of Interactive Systems based on Brain- Computer Interfaces
}

\author{
Alessandro L. Stamatto Ferreira, Leonardo Cunha de Miranda, Erica E. Cunha de Miranda, Sarah Gomes Sakamoto \\ Department of Informatics and Applied Mathematics \\ Federal University of Rio Grande do Norte (UFRN) \\ Natal, RN, Brazil \\ alexmatto@ppgsc.ufrn.br, leonardo@dimap.ufrn.br, erica@dimap.ufrn.br, sarahsakamoto@ppgsc.ufrn.br
}

\begin{abstract}
Brain-Computer Interface (BCI) enables users to interact with a computer only through their brain biological signals, without the need to use muscles. BCI is an emerging research area but it is still relatively immature. However, it is important to reflect on the different aspects of the HumanComputer Interaction (HCI) area related to BCIs, considering that BCIs will be part of interactive systems in the near future. BCIs most attend not only to handicapped users, but also healthy ones, improving interaction for end-users. Virtual Reality (VR) is also an important part of interactive systems, and combined with BCI could greatly enhance user interactions, improving the user experience by using brain signals as input with immersive environments as output. This paper addresses only noninvasive BCIs, since this kind of capture is the only one to not present risk to human health. As contributions of this work we highlight the survey of interactive systems based on BCIs focusing on HCI and VR applications, and a discussion on challenges and future of this subject matter.
\end{abstract}

Keywords-Brain-Computer Interface; BCI; Headset; EEG; Human-Computer Interaction; HCI.

\section{INTRODUCTION}

The evolution of technology provides significant changes in the way users use interactive systems. With the everincreasing usage of tablets and smartphones, it can be observed that interaction between users and applications will take place through smaller displays and touchscreens. Whereas modern controls such as WiiMote and Kinect highlight the need for interaction adjustment considering user physical movements in their context of use to support appropriate utilization of systems. Therefore, design and development of interactive systems should follow new trends of technologies in order to provide better user experience, increasing productivity and offering intuitive actions for execution of different tasks.

With technological advancements different kinds of interaction which use our bodies have emerged, enabling the use of various body parts other than our hands. For example, Harrison et al. [10] demonstrated the possibility of using human skin as a touch interface, Nam et al. [24] presented a wheelchair controlled by tongue movements, and Liu et al. [17] proposed an eye-tracking system as well as several examples of eye-tracking for human-computer interaction. Also, Vernon and Joshi [31] propose using a muscle above the ear - which lost its function along with human evolution - to control a television. However, it is possible to go further and use a part of human body's central axis, already presented in all forms of human interaction: the brain.

Brain-computer interface is a research field been studied since middle of 70s in diverse areas of knowledge such as neuroscience, biomedicine, automation and control engineering and computer science. Meanwhile only recently cost and accuracy required for civilian use have been achieved. People with severe motor impairments are main beneficiaries of braincomputer interface researches, as persons with locked-in syndrome, i.e. a rare condition characterized by paralysis of voluntary muscles except for the eyes. Nevertheless, we realize that people without any disability are also potential users of solutions which promote interaction between humans and computers through cerebral signals, in the most possible natural way.

However, interactive aspects of BCIs remain poorly explored by researchers, probably due to the intrinsic complexity of areas involved in this research topic. New studies are coming out guided by some computing areas such as Human-Computer Interaction (HCI) and Virtual Reality (VR). A prime example is a work by Solovey et al. [30], which describes the use of brain-computer interaction in a multimodal interface. Also, Friedman et al. [49] present a braincomputer interface with virtual reality. There is therefore a strong need for a detailed study to identify clearly and objectively current limitation of brain-computer interface from an interactive perspective.

Millán et al. [22] present a brain-computer interface review focused on motor substitution with neuroprostheses and recovery through neurorehabilitation. The authors discuss brain-computer interface applications in an assistive technology context, such as using sensors in a wheelchair for better brain-computer interface control. Lotte et al. [50] review and explore brain-computer interface works that use VR, focusing on the design of brain-computer interface based on VR applications. In this paper, we discuss brain-computer interface solutions in an interactive perspective, such as evaluation of user's cognitive workload and the lack of freedom regarding visual attention. Furthermore, our work focus on HCI and VR aspects, while taking in consideration 
both healthy and impaired users. Afterwards, we identify and discuss several challenges in this context.

This paper is organized as follows: Section II contextualizes brain-computer interface area, introducing basic concepts and technologies; Section III presents a survey of brain-computer interface; Section IV presents several challenges related to brain-computer interfaces; Section V discusses this research topic; and Section VI concludes the paper.

\section{BRAIN-COMPUTER INTERFACE}

Brain-Computer Interface (BCI) is a mode of interaction between human beings and computers which does not use any muscle, since system is controlled through user's mental activity captured with specific equipment. According to Wolpaw et al. [34], BCI is a communication system with two adaptive components that mutually complement each other. For these authors, at the current technology stage, users should fit into BCI to control the system since it should adapt itself to user's mental signals. Hence, user must understand the system which must adjust itself to user, both required for BCI to succeed.

BCI requires reception of brain signals captured directly from human brain. There are three different ways to capture these signals, i.e. (i) invasive, (ii) partially invasive, and (iii) noninvasive. Invasive capture is characterized by introduction of implants into user's encephalic mass, directly into the gray matter, providing high quality signal reading; however it causes great inconvenience and risks to human health. In partially invasive capture, implants are placed beneath the skull without drilling the brain. Despite its lower quality signals, this signal capture form presents lower risks to health as compared with invasive approach. Lastly, noninvasive capture enables gathering information without any implant since sensors are placed on the scalp, fully external to the body. Noninvasive BCIs are more convenient and easy to use, and due to technological advancements of current solutions, provide good quality signal capture. It is also the only one to not present risk to users' health. For this reason, this paper focuses only on noninvasive BCIs.

There are three most common techniques to obtain cerebral information, i.e. (i) electroencephalogram (EEG), (ii) functional magnetic resonance imaging (fMRI), and (iii) functional near-infrared spectroscopy (fNIRS). With EEG, brain activity is captured through sensors called electrodes. It is possible because neurons communicate with each other via electrical signals, which eventually reach brain surface and then are captured by electrodes. The fMRI technique measures brain activity through blood oxygenation and flow, which increase in the specific area involved in mental process. This capture technique requires usage of equipment with considerable dimensions and a scanner with a large magnetic field. And fNIRS method also measure brain activity through blood oxygenation and flow, but it is based on identifying variation of optical properties in brain images. Near-infrared light is sent into the user's forehead and, through light detectors, the reflected rays are picked up and correlated to specific concentration of oxygen.
Fig. 1 shows different equipment to gather cerebral information in a noninvasive way with the above mentioned techniques, i.e. EEG electrodes capturing electrical signals (Fig. 1a), scanner fMRI with magnetic resonance imaging (Fig. 1b), and spectroscopic sensors with near-infrared radiation (Fig. 1c). Nowadays, only EEG and fNIRS enable to gather cerebral information in a real usage scenario due its relative low-cost and portability. Moreover, EEG has the best temporal resolution, which means that it captures signals faster than others, and hence this method is the most used in BCIs.

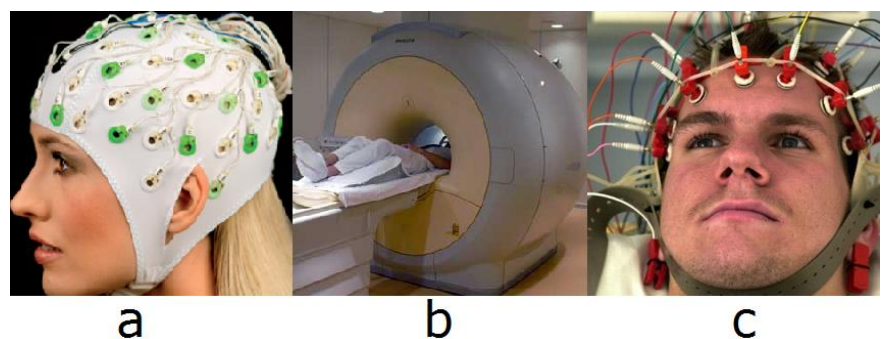

Fig. 1. Nonivasive equipments used to capture cerebral information (a) EEG electrodes (b) fMRI scanner (c) spectroscopic sensors. Sources: $[1],{ }^{1}, 2$.

There are different capture devices, which vary greatly in shape and may be a cap, tiara, headband, helmet, or even loose electrodes. In this paper we unify all these terms in a single one: headset. Hence, we consider as headset a set of sensors placed on user's head. For marketing purpose, companies have been developed more portable headsets with attractive designs at lower costs. These devices aims to provide greater comfort as compared with equipment showed in Fig. 1. In 2009, NeuroSky ${ }^{3}$ has launched MindSet, a wireless headset with a single EEG electrode and capable of measuring user concentration. This company has others headsets available such as MindWave, launched in 2011. In 2009, other company named Emotiv ${ }^{4}$, launched EPOC: a wireless headset in a tiara format. EPOC has $14 \mathrm{EEG}$ electrodes and a gyroscope, which measures head movements. BCI researches with fNIRS in real usage scenarios usually use sensors covered with a headband in order to maximize comfort. Fig. 2 shows some BCI headsets, i.e. NeuroSky MindSet (Fig. 2a), Emotiv EPOC (Fig. 2b), and fNIRS sensors covered with a headband (Fig. 2c).

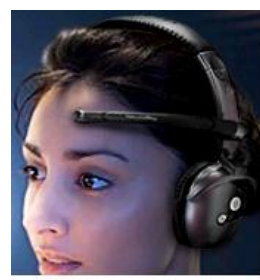

a

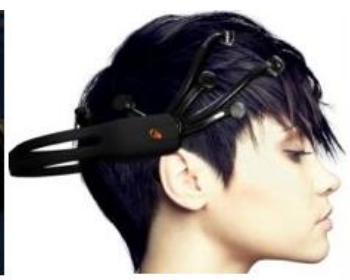

b

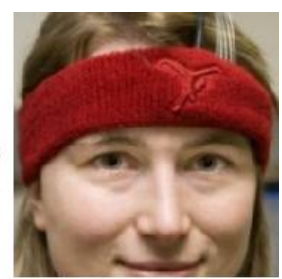

C
Fig. 2. BCI headsets (a) NeuroSky MindSet (b) Emotiv EPOC (c) fNIRS snsors covered with a headband. Sources: [7],[1],[29].

\footnotetext{
http://blogs.oem.indiana.edu/scholarships/index.php/2009/10/26/neurons-an d-electrodes/fmri_groot/.

${ }^{2}$ http://www.spiegel.de/fotostrecke/fotostrecke-13782-3.html.

${ }^{3}$ http://www.neurosky.com.

${ }^{4}$ http://www.emotiv.com.
} 


\section{A. Thoughts Recognition}

BCIs require recognizing a thought or mental activity in order to activate an action. An ideal scenario should be to think about turning a lamp on and then, BCI system recognizes this thought and turns a lamp on automatically. Currently, recognizing specific thought such as "turn lamp on" is still very difficult. However, there are three mental activities recognized with certain precision, which are commonly used on BCIs applications, i.e. (i) concentration, in which Alpha and Beta waves are used to estimate user's attention and relaxation/meditation, (ii) stimulus response, in which brain responses are detected when user focus on certain flashing graphic elements (visual stimulus) and/or special sound patterns (sound stimulus), and (iii) imagined movement, in which is possible to detect kinetic thoughts, such as imagining your right hand opening and closing, due to the synchronization and desynchronization of $M u$ rhythm.

The detection for a stimulus response (ii) is subdivided into two types, i.e. oscillating stimulus and transient stimulus. In oscillating stimulus, elements are differentiated by frequency, such as LEDs where each one flashes - oscillates in a different frequency, inducing a natural response from brain and generating electrical activity in the same or multiple frequency of stimulus. Other example of oscillating stimulus is in the case of two sounds from different frequencies, which generates a specific response to the focus in each one. The response for a visual oscillating stimulus is called Steady State Visually-Evoked Potential (SSVEP) and a response for a sound oscillating stimulus is called Steady State Auditory Evoked Potential (SSAEP). Transient stimulus are differentiated by response to a transition from a visual/sound state to another. When an individual waits for a certain stimulus among other similar stimulus, a wave called P300 is generated as a response. An example would be five squares, off most of the time, which each one turns on for a short time and turns off again. User concentrates in one square and when this square turns on, a P300 wave is generated due to the small "surprise" caused by the transition from off to on. Likewise, it is possible to identify a user's response when listening a sound repeatedly and then suddenly, a different sound occurs.

One of the first visual stimulus-based applications commonly used in tests is typing, called speller. In this test, screen contains letters from ' $A$ ' to ' $Z$ ' arranged in a grid/matrix and user must concentrate in a specific letter. BCI recognizes which letter and presents it to user. Then, user can focus and concentrates in other letter and therefore, letter by letter to form words. In SSVEP-based BCIs, each letter flashes intermittently in different frequencies, whereas in P300-based BCIs, one line/column flashes at time in a random pattern. When the line corresponding to the user's chosen letter flashes, P300 wave is recognized, indicating that user is focusing on that line. Likewise, when the column flashes this wave is identified. Thus, with both the line and column recognized, it is possible to identify the letter chosen by user. Fig. 3 shows two different spellers which enables word entry like as a keyboard. In Fig. 3a the speller is used on P300-based solutions and Fig. $3 \mathrm{~b}$ illustrates a speller for SSVEP-based solutions.

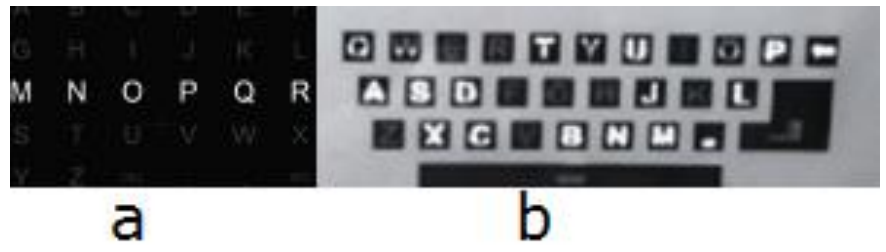

Fig. 3. Spellers based on (a) P300 (b) SSVEP. Sources: [26],[13].

In BCIs, processing can be performed online and offline. Online processing occurs in real time while the user utilizes a BCI and offline processing is performed after user experiment with a post-processing approach in order to obtain the maximum precision. There is also a BCI classification regarding rhythm, i.e. synchronous and asynchronous. In synchronous BCIs, commands are interpreted at a constant time rate. Therefore, after every certain amount of time, a command is recognized regardless of user's intent. Whereas asynchronous BCIs - also called self-paced - give control to the user to recognize a command only when wanted.

We consider pertinent presenting some fundamental concepts of the BCI area in order to provide a refined understanding about the literature works presented in this survey. The objective of this background is to present a theoretical overview, not an introduction tutorial about BCIs. The following section presents a survey that comprises a relevant part of the literature about this research topic.

\section{SURVEY}

The survey presented in this paper describes works which address new BCIs. These interfaces are related to daily task accomplishment, now possible through cerebral waves. Therefore works presented demonstrate the potential of new interaction forms with interactive systems through BCIs. Moreover, to a greater identification of proposes and challenges of this research area we grouped works according detection approach it utilizes, i.e. (a) visual stimulus response, (b) sound stimulus response, (c) concentration, (d) imagined movement, and (e) neurofeedback.

The search strategy consisted of automatic and manual searches in scientific libraries and bibliographic databases. Automatic search was conducted in IEEE Xplore, ACM DL, Springer, Elsevier, Scielo, Scopus, ISI Web of Knowledge, and also in Google Scholar; manual search was made in PLoS Biology and Frontiers in Neuroscience Journals. In the search process we used a combination of the following keywords in English (presented in this paper in alphabetical order): BCI, brain, computer, HCI, human, interaction, interface, reality, virtual, and VR. For this study we select only recent works, i.e. paper published in the last five years.

\section{A. Visual Stimulus Response}

Mauro et al. [21] exhibit the use of a BCI to control the cursor of a desktop operating system. They implemented two BCIs based on P300, one exogenous and other endogenous; in an exogenous interface the user focus is external, while on an endogenous interface the user focus should be at the center (internal). Both interfaces allow four movement directions with 
four squares - one in each side - representing position objectives for testing purposes. On the exogenous interface, those squares flash, and the user has to focus on the square in desired direction. The squares do not flash in the exogenous interface. Instead, one letter is showed on the center of screen, alternating between the initials of Italian words for directions, i.e. alto (up), destra (right), $\underline{b} a s s o$ (down), and sinistra (left). The user has to count the occurrences of the letter representing the desired direction. Eight patients, half healthy and the other half in advanced state of paralysis, participated in the experiment. Results demonstrated that there was no difference on the precision of the healthy group versus the paralyzed one, which indicates that the interfaces devised do not depend on motor abilities.

Hood et al. [42] developed a BCI control for a car driving virtual simulation using CARRS-Q. CARRS-Q simulator consists of a platform with 180 degrees frontal projection and three simulated mirrors. The BCI uses three LEDs as SSVEP stimulus, each one offering a configurable command. One possible configuration would be with three LEDs, i.e. one to right steer the wheel, another one for left steering it, and the last for straight steering. It reached good precision rates but still cannot be applied in real situations, because it is not safe enough. In the authors opinion, a virtual ambient will be essential for improvements on the interface and safety guarantees of future car-driving BCIs. Fig. 4 showcases the system being used.

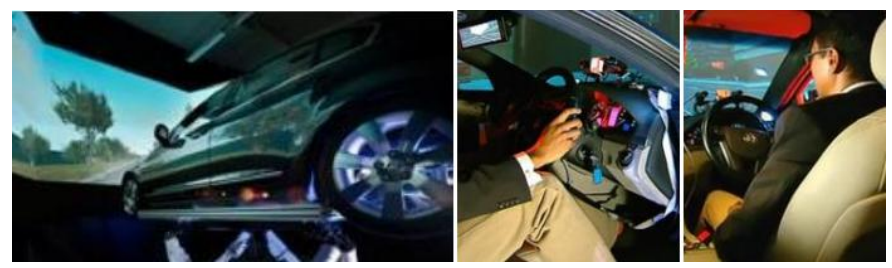

Fig. 4. BCI for car driving in virtual simulator CARRS-Q. Source: [42].

A different BCI application is a web browser created by Mugler et al. [23]. The BCI is based on the P300 and follows Mankoff et al. [20] web usability needs. For Mankoff et al. [20] all web browser systems must offer the following features: web navigation, page navigation with fewest commands possible, history browsing, bookmarks, and text input. In addition to those ones, the authors add functionalities like an URL bar. Speed is considered an interesting factor but not a need. Another BCI web browser is the one created by Xu et al. [35], which uses the SSVEP approach. The application allows searching in Google and inputting text. The authors mounted a hardware, in which a small LED board with six LEDs is attached on a conventional notebook. Each LED represents an option and these LEDs are used to input characters, to navigate a $6 \times 6$ menu, and to select web browsing commands like "next URL" and "HOME". The interface achieves great precision $(92 \%)$ but it is very slow (four and a half minutes for a simple Google search). Although the described application is interesting, Liu et al. [18] argue that SSVEP BCIs have a fatigue factor due constant and intermittent flashes. For this reason, these authors created an alternative BCI. Like the usual SSVEP, the interface uses a visual stimulus differentiated by frequency and also a movement frequency instead of the usual light frequency. According to the authors, this new SSVEP interface offers an increased comfort with a good precision $(83 \%)$.

Ceccoti [4] developed an asynchronous BCI speller based on SSVEP. The speller objective was to achieve an intuitive system where even inexperienced users could successfully use it, while causing the least possible discomfort. Therefore, the letters are divided into groups and what flashes is the contour of those groups. The system automatically configures the BCI and the asynchronous nature of the interface leaves the user more relaxed. Another interesting work is the one presented by Campbell et al. [2] that proposes the NeuroPhone, a iPhone application in which phone calls are made through a BCI using the EPOC. A contact photo grid is showed to the user and one photo flashes at time in a P300 fashion. Then, the user focuses on the contact to call. Fig. 5 shows the process of calling a contact.
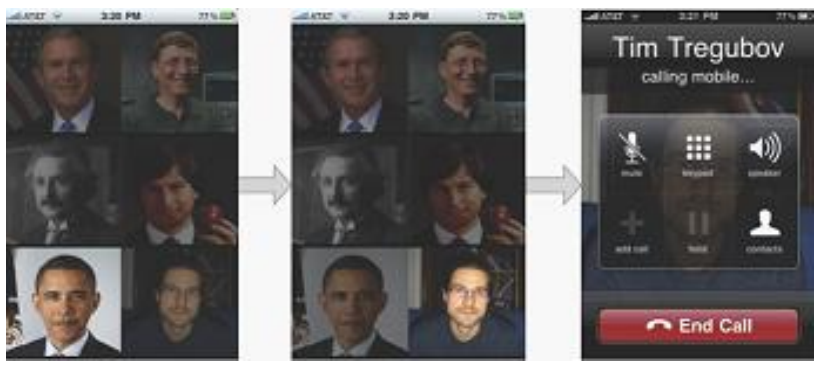

Fig. 5. Contacts flash, one by one, detecting the user visual focus. A phone call is made to the one choosed. Source: [2].

A remote robot is controlled in the SSVEP BCI made by Gergondet et al. [6]. In this system, a real-time video displays the robot "vision", i.e. a camera coupled on the robot. On the controlling machine - notebook - the interface mixes the robot vision with four red squares at the four sides - top, bottom, left, and right - that act as the visual stimulus. Focusing on one of these directions, the robot increases speed in the same direction. For testing purposes the authors use a robot benchmark known in the robotic area as SLALOM, the robot BCI successfully passes in the benchmark test. Yuksel et al. [37] employ a P300 BCI for object selection. These objects are arranged in a multi-touch table display and a computer vision algorithm computes the approximated shape for each object. This shape is expanded and flashed under the object following the same process in P300 spellers with an object grid instead of a letter grid. Fig. 6 exhibits the BCI table in action.
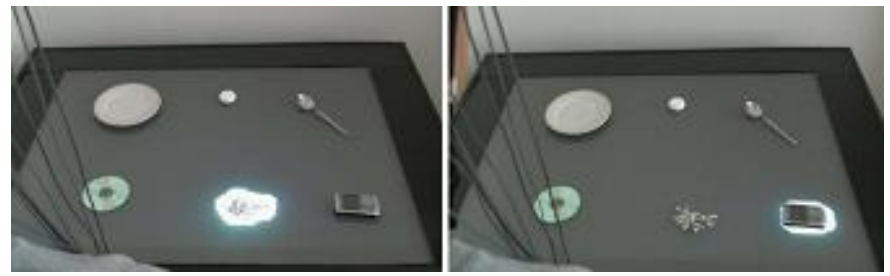

Fig. 6. Multitouch display interface, where objects are enlightened one by one, triggering a P300 wave. Source: [37].

Grierson e Kiefer [7] test MindSet - conventionally used to detect concentration - for a BCI based on P300. The efficacy was benchmarked through an experiment, in which two squares - one blue and one red - take turns flashing and the 
$\mathrm{BCI}$ has to detect which one the user is focusing. With squares of same size the precision is good $(78.5 \%)$, while with squares of different sizes the precision is close to $100 \%$. The results indicate that it is possible to use a commercial headset for BCI applications based on visual stimulus. Wang et al. [33] developed a smartphone BCI application based on SSVEP for calling contacts by number typing. The interface contains numbers 0 to 9 , a confirmation option (Enter), and a correction option (Backspace). Frequencies between 9 and $11 \mathrm{~Hz}$ are used as visual stimulus. Normally those frequencies cannot be achieved in a smartphone due to screen refresh rate but the authors employ a special technique of black/white alternating patterns to circumvent this restriction. A good precision was achieved (close to $100 \%$ ).

Hakvoort et al. [9] implemented a SSVEP BCI game, whose goal is to lead sheep into a sheep pen by controlling shepherds dogs. Kapeller et al. [14] remark the importance of analyzing BCIs in a distracting context. They did a benchmark where a user has to focus on SSVEP visual stimulus, overlaid on a movie. The experiment showed that some precision $-6 \%$ on average - is lost in this distraction context. One of the participants had a very large loss of precision (40\%) and according to the authors this indicates that some users are more sensible to visual distractions.

Escolano et al. [45] developed a BCI telepresence system, wherein a robot is remotely controlled from a different geographic location. A camera on top of the robot shows his current "vision". The BCI is based on the P300 and the graphical interface imposes options as augmented reality on the robot vision with commands like "turn left" appearing as icons. Besides the camera, the robot is also equipped with a laser sensor, wheelchair and a location tracker based on measurements of the rotations of the wheel. The BCI developed has two operating modes: robot navigation, where a point grid is used as Visual Stimulus for choosing a destination and camera exploration, in which the point grid is used to indicate where the camera should look at. Five users participated in an experiment, having to navigate the robot through close space situations, all users managed to control the robot successfully to the final position.

An assistive BCI application is the one by Grigorescu et al. [46], where the interface controls a robotic assistant for helping people with motor deficiencies. The system is named by the authors as FRIEND (Functional Robot with dexterous arm and user-frIENdly interface for Disabled people). The BCI was an addition to a new generation of the robot system to supply quadriplegic needs. FRIEND combines several modules, i.e. wheelchair, robotic arm with gripper, EEG headset, monitor and camera (used for machine vision). The BCI uses the SSVEP approach, where five LEDs act as visual stimulus, each one representing a menu option. FRIEND is a semiautonomous robot and has two modes, i.e. (i) complete autonomous mode, wherein objects are automatically recognized and manipulated, and (ii) shared control mode, where the user assists the system with ambient information, like approximate object positions. System performance is measured in four scenarios: prepare and serve a drink, prepare and serve a meal (showed in Fig. 7), tasks at a library service desk, and tasks for keyboard maintenance (checking if they're working correctly). Those tests indicate that Friend still needs improvement for real world usage but it has great potential.

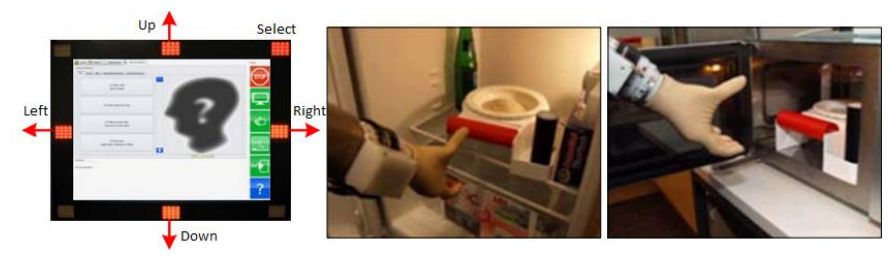

Fig. 7. A user selects the "prepare meal" in the FRIEND BCI system. Source: [46].

Kaufmann et al. [15] developed the Optimized Communication System, a P300 BCI speller where a single button automatically configures and adjusts the system. The user presses the button one time to start EEG signal capture, and a second time to stop the capture and use the data collected to configure and calibrate the system. It also improves on other spellers in information transmission speed through word auto completion - the word prevision was written in Python -, the complete words appear together with the letters in the grid. Following the idea of less configuration as possible, the application creates the word base automatically - navigating the web and computing word frequencies for a determined idiom. Poli et al. [41] take BCI domain to space interaction, creating an spaceship navigation BCI controller. BCI usage on space applications have great potential since they allow piloting, collaboration and machine control without the, in several situations restrict, movement of hands. The developed BCI uses P300 approach with an innovative graphical disposition. Eight gray circles form a greater circle and one by one is flashed to red or green - randomly selected - color. Three volunteers participated in a simulation, whose goal was to make a path with the ship, passing as close as possible to the sun. The authors also devised a cooperative mode, in which the control is made simultaneous by more than one person. On the experiments the cooperative mode had better precision than the single-person mode. Still, the BCI developed has to improve to achieve high-enough precision for real world usage. Nevertheless, it is an excellent result and offers an optimistic vision for future BCI space applications.

Edlinger et al. [43][44] developed a domotics BCI application based on an hybrid approach, using SSVEP for turning on and off the application, while using P300 for command selection and activation. They define four requirements for domotics $\mathrm{BCIs}$, i.e. (i) signal amplifiers most work even on noisy environments, (ii) EEG capture has to be made with a portable device, to avoid collisions and user irritations, (iii) for real time experiments it's necessary to connect the BCI to a virtual reality simulation, and (iv) the communication interface between BCI and VR needs to offer a satisfactory degree of freedom. In the VR the user is equipped with 3D glasses and a head-position tracker. EEG signals are captured through g.MOBIllab+ amplifier, those are send to a PC which controls the virtual ambient using XVR (eXtreme VR). Video output is projected into a high resolution surface (powerwall). The virtual ambient is composed of three rooms, each one with controllable devices like television, music player, telephone, lights and door. Commands are divided into seven categories: lights, music, telephone, temperature, 
television, move and "go to". Each of those categories act as an interface "mask", one screen that has only the relevant commands for that category. Fig. 8 shows the BCI and controlled virtual ambient.
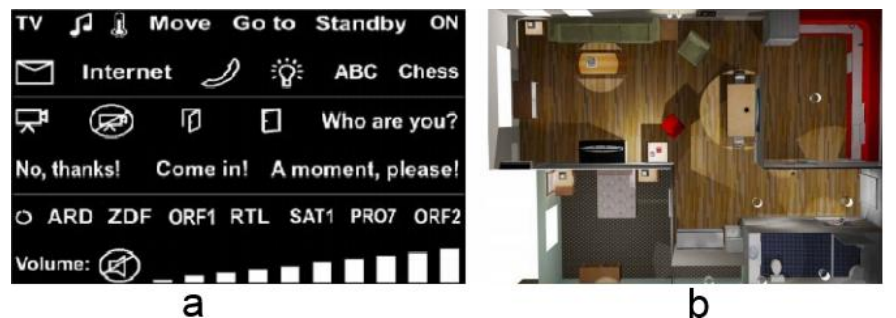

Fig. 8. A (a) P300-based BCI for controlling (b) a virtual domotics environment. Source: [44].

\section{B. Sound Stimulus Response}

Lotte et al. [19] developed a sound stimulus based BCI using a P300 approach. Their objective is to present a BCI efficient in a scenario of great mobility, where concentration in a visual stimulus is a hard task and user movement can cause interference on EEG capture, since movement is responsible for a great part of brain electrical activity. The BCI developed uses two sounds as stimulus, one rarely appears - a ring bell sound ("Ding Dong"), while the other sound frequently plays a buzzer sound. The user has to focus on the ring bell sound, counting the number of occurrences, eliciting a P300 wave each time the sound plays. They conducted an analysis to identify movement interference on P300 detection and three movement states were tested, i.e. sitting, standing and walking. Experiment results were promising: no significant precision was lost due to movement interference and a good EEG capture was possible in all states tested.

On the work of Kim et al. [16] a BCI based on steady state evoked potential was conceived. However, sound frequency (SSAEP) was used instead of the usual visual flashes frequency (SSVEP). Two sounds of different frequencies are used, each one in different sides of the user - left/right - to strengthen contrast between them. A good precision was achieved, 71\% online and $86 \%$ offline. One disadvantage of such sound based BCIs is the binary choice, i.e. the user can only choose between two options (sounds). Hill and Schölkopf [12] research ways to improve sound based BCIs. They use the same approach of SSAEP - sound frequency - combined with spatial location - one on the left of the user, the other on the right - in a way that resembles the "surprise" associated to P300 approaches. Their BCI achieves higher performance, obtaining $85 \%$ precision online.

\section{Concentration}

Coulton et al. [5] created a smartphone game named Brain Maze, which uses BCI as one of the controllers using the MindSet. The game objective is to move a ball from a start point to a finishing destination. Moving the ball is done through accelerometer, but some obstacles need to be overcome using a BCI. Some paths are blocked by closed gates. There are two types of gates, i.e. attention and meditation gates. The user has to increase concentration to open attention gates, while he needs to relax to open meditation gates.

Marchesi [40] presents a BCI prototype for interactive cinema, the Neu system. Neu system measures the user degree of concentration/relaxedness using a MindWave and those measurements affect course of events in the history of the interactive movie. Neu is an evolution of MOBIE System, developed by the same author. Mobie monitors and records the degree of concentration/relaxedness of the user while he watches a movie. From this feedback the user engagement in each scene is obtained. Neu takes this concept one step beyond offering, in the authors' viewpoint, a BCI interactive, immersive, and personal experience.

\section{Imagined Movement}

Poor et al. [27] assess EPOC capacity in a BCI based on the imagination of kinetic actions. On the experiments the objective was to rotate a cube after an initial brain signal recording and calibration. The precision was low (59\%) but the authors attribute the cause to training lack and immaturity of Stimulus-Less BCI systems and techniques. Friedman et al. [49] conduct research on navigation in a cave virtual environment based on imagined movement. The VR consists of a street with people spread out and stores on the sides - those can be projected in stereo view shutter glasses for increased realism and experience. To walk, the user has to imagine the user feet moving, while head rotation - tracked through an accelerometer - is used for changing walking direction and imagined hands movement - to pass the impression of "touch" - is used for interacting with other persons on the street. The virtual people remain at still until the user interacts with them. In that instant, they start to walk to indicate interaction success.

One of the first authors' experiments [48] was conducted with a quadriplegic patient, who successfully moved across the virtual environment as displayed in Fig. 9. Another experiment [47] with 10 participants compared BCI precision in two different scenarios: a controlled choice scenario and a free choice scenario. In the first one, user receives a sound cue for the action he must realize and in the other one, user freely chooses which action to perform. The precision on the controlled choice scenario was higher $(82.1 \%)$ than the free choice scenario (75\%), which presents a challenge to be overcome, since freedom of choice is essential in interactive systems.
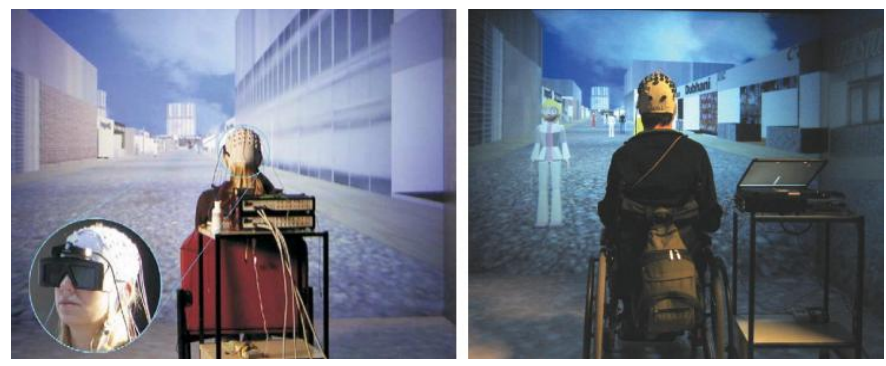

Fig. 9. User walks in a virtual street imagining feet movement. Source: [48].

Leeb et al. [39] leverage a conventional game - i.e., which was not originally designed to work with a BCI controller named PlanetPenguin Racer. In the original game a penguin is 
controlled to descend a snow mountain, collecting fishes on the way. The game was modified to float all the fish, suspending them mid air. Jumping is the only way to catch the fish in this new version and to jump, user has to imagine feet movement. For increased immersion the game happens on a cave virtual reality, where the user is surrounded by walls with projectors directed to each one of them. As such, the game uses a multimodal interface: the penguin's direction is controlled by a joystick and the jumps by BCI. An experiment with 14 users demonstrated that concomitant use of joystick with BCI did not decreased BCI precision. Furthermore, leg positioned sensors proved that jumps - BCI control - did not use any muscles. A pure joystick control achieved highest precision on catching the fish, as expected by the authors. However, the majority of users preferred the BCI controls, remarking the fun of jumping only through mental power. According to authors, the game needs only a short time of training, being entertaining without leaving the player bored with long sessions of training.

\section{E. Neurofeedback}

Vi and Subramanian [32] were able to detect an electrical potential called Error-Related Negativity, caused by user frustration when an interaction does not occur as planned. An example is when the user tries to select an option among others but misses, by user or system error, and chooses one he did not want, getting the user frustrated. A BCI detecting this frustration - through Error-Related Negativity potential - a system could try to auto-correct the interaction error, choosing the closest option of the one miss-selected. An experiment was made to measure the precision of successfully detecting this potential. The authors choose to analyze the precision through an interaction test known as Superflick, where a user has to "throw", with a drag-and-drop movement, a small ball into a big target ball. If the user misses the target the system must auto-correct the interaction, trying to achieve a most satisfactory state for the user. The BCI application conceived achieved $70 \%$ precision, and proved that is possible to detect interaction errors, and use this information to provide a better experience for the user, compensating the error with an action rollback, giving a small advantage to a player, or selecting close objects. Fig. 10 shows a user participating on the Superflick test, "throwing" a ball while the headset captures the user frustration.

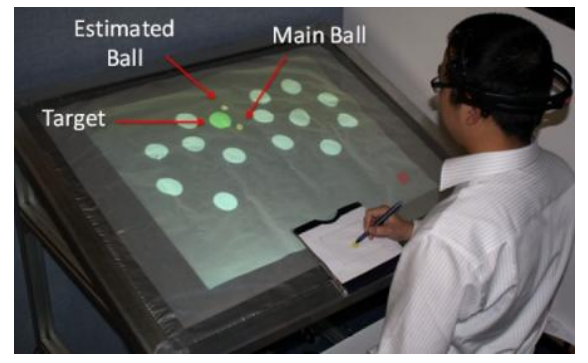

Fig. 10. Superflick interaction test using a BCI for auto-correction. Source: [32].

Solovey et al. [30] created a BCI application - Brainput that avails the priority of tasks being done by the user. This priority is estimated through detection, using fNIRS sensors, of three mental states of concurrency, i.e. (i) branching, when the current task is interrupted, and replaced, by another of increased priority, (ii) delay task, where a user receives another task but chooses to ignore it, implicitly indicating that few resources must be allocated to that lower priority task, and (iii) dual task, where the user works on two tasks of same priority, and constantly switch between them. The BCI system conceived by the authors adapts to allocate more resource to tasks of higher priority. For testing this BCI an experiment was made with 11 users, they needed to remotely control two robots, one blue and one red. They could only control one robot at time, switching between them. Both robots expanded resources to move, and had to reach a specific destination and send a signal. A priority was assigned to each robot, and performance was compared between a Brainput interface doing the robot control switch, and an interface where the robot where more autonomous. The results showed the Brainput interface as the better one.

\section{F. Summary}

Table I summarizes 29 interactive systems based on BCI presented above and grouped in this table by year of publication. As previously described, BCIs go beyond of computer control, comprehending domains such as domotics, assistive interfaces, robot control and electronic games. It is important to highlight that most of stimulus-based BCIs uses a visual form due to its higher precision. Table I shows approach used by BCI classifying works in (V)isual stimulus, (S)ound stimulus, (C)oncentration, (I)magined movement, and (N)eurofeedback.

\section{TABLE I.}

LITERATURE WORKS PRESENTED IN THIS SURVEY.

\begin{tabular}{|c|c|c|c|}
\hline \multicolumn{2}{|c|}{ Work } & \multirow{2}{*}{$\begin{array}{l}\text { Detection } \\
\text { approach }\end{array}$} & \multirow{2}{*}{ Brief description } \\
\hline Year & Ref. & & \\
\hline 2007 & [48] & $\mathrm{I}$ & Avatar control/walking in a virtual street \\
\hline \multirow{2}{*}{2009} & [35] & $\mathrm{V}$ & Web browsing and speller \\
\hline & [19] & $\mathrm{S}$ & BCI control while walking \\
\hline \multirow{7}{*}{2010} & [23] & V & Web browsing \\
\hline & [18] & $\mathrm{V}$ & Web browsing and speller \\
\hline & [4] & $\mathrm{V}$ & Asynchronous speller \\
\hline & [2] & $\mathrm{V}$ & Call a contact through smartphone \\
\hline & [37] & $\mathrm{V}$ & Object selection in a real ambient \\
\hline & [33] & $\mathrm{V}$ & Phone dialing \\
\hline & [49] & $\mathrm{I}$ & Avatar control/walking in a virtual street \\
\hline \multirow{8}{*}{2011} & [21] & $\mathrm{V}$ & Mouse control \\
\hline & {$[6]$} & $\mathrm{V}$ & Robot control with real time vision-camera \\
\hline & [7] & $\mathrm{V}$ & Headset benchmark \\
\hline & [9] & $\mathrm{V}$ & Sheep game \\
\hline & [43] & $\mathrm{V}$ & Domotic control in a virtual house \\
\hline & [16] & $\mathrm{S}$ & SSAEP benchmark \\
\hline & [5] & $\mathrm{C}$ & Smartphone Maze Game \\
\hline & [27] & $\mathrm{I}$ & Commercial headset benchmark \\
\hline \multirow{9}{*}{2012} & [45] & $\mathrm{V}$ & Telepresence robot with vision-camera \\
\hline & [46] & $\mathrm{V}$ & Auxiliary robot for disabled people \\
\hline & [15] & $\mathrm{V}$ & No configuration speller with word prediction \\
\hline & [44] & $\mathrm{V}$ & Domotic control in a virtual house \\
\hline & [42] & $\mathrm{V}$ & Car control on virtual CAVE simulation \\
\hline & [12] & $\mathrm{S}$ & SSAEP + P300 benchmark \\
\hline & [40] & $\mathrm{C}$ & Interactive movies \\
\hline & [32] & $\mathrm{N}$ & Superflick interaction test \\
\hline & [30] & $\mathrm{N}$ & $\begin{array}{l}\text { Resource control through brain concurrency } \\
\text { detection }\end{array}$ \\
\hline \multirow{2}{*}{2013} & [41] & V & Spaceship control in a virtual simulation \\
\hline & [39] & I & CAVE VR game \\
\hline
\end{tabular}




\section{Challenges}

Through reflection on the BCI literature review, we identified several challenges with implications for user interaction. These challenges must be faced in order to BCIs been used in a more effective manner with interactive systems. To a better understanding, we grouped challenges in topics.

Most of existing BCIs causes a high level of fatigue, demanding high concentration or attention to quick and intermittent stimulus. In addition to fatigue inconvenience, BCI may not work since user cannot reach enough level of concentration. In [11], Hasan and Gan try to assure BCI's operation even when user is tired. The BCI implemented by these authors properly monitors user performance and when it declines, system activates an adaptation which reduces the concentration limit necessary to interact with system. The use of VR in BCI applications may assist in this process, providing a high immersive environment. It motivates the user while interacting with the system, consequently increasing users' attention and concentration levels.

Concentration required to stimulus also causes a mixture between input and output since mental activity is being constantly monitored and user's focal point changes the input. Instead of relax, user must concentrate on a point as input and look to the output. For example, a user watching a movie; the user has to look at a specific point on screen instead of a part of scene that the user wants to see. At this stage, interaction has a forced aspect, instead of natural aspect presented in the case of user may decide which region of visual output the user wants to focus. A similar challenge occurs with traditional interactions since interaction flux often depends on user perceiving certain feedbacks, mainly the ones issued by computational system. In VR environments this issue is exacerbated since the lack of visual freedom may disrupt the immersion. This challenge is also applied in other 3D environments such as augmented reality, which may bring other problems such as safety since user has to focus on a certain point and may not pay attention in what comes ahead.

According to Wolpaw et al. [34], with current BCI technologies, users must fit themselves into the system in order to control it; speed and satisfaction of this adjustment depends on system's intuitiveness. During tasks accomplishments on applications, the study of user's visual focus and intuitiveness of graphical interface are conducted by HCI researchers. These researchers must apply $\mathrm{HCI}$ techniques in BCI context in order to develop a visual interface with as fewer nuisances as possible regarding constant transitions of user's visual focus and also easy-to-use, providing a fast user's adjustment to the system.

Using a BCI system is often a complex task. It is necessary to verify electrodes' position on user's head and configure different parameters before using the system. Furthermore, users must know which technology is best suited to their needs, including purpose and profile. Randolph [28] evidences that factors such as gender, caffeine and experience on videogames or musical instruments, affect mental states and waves, which are tracked by capture technologies. Hence, different people may have different needs regarding BCIs, which makes the use of this kind of interaction in a practical way even more difficult. 3D environments technologies are also impacted by this challenge since technology which provides the best user experience may vary for each user. So, when mixing both 3D and BCI technologies, user's needs must be considered carefully.

In most cases BCIs do not provide mobility to users. Users must obligatorily remain at still and quiet, preferably sitting down, during test application. However, in a real using situation user may need to use BCI while the user walks on the street in order to control a smartphone, for example. In addition, BCIs must also provide comfort to user. An EEG headset must be easy to carry on and simple to use on daily routine, as well as a person uses a headphone to listen to music. An EEG headset must be lightweight, not only to provide mobility but also to enhance use experience; its weight must not be uncomfortable to user. Other significant inconvenience is caused by a gel, which is applied on electrodes in most of EEG headsets in order to enable signal capture, even though Guger et al. [8] indicate that dry electrodes are priority to user. To meet this requirement, authors present a non-commercial EEG headset with dry electrodes and high precision.

Another similar challenge is the possible conflicts between different interface devices, i.e. using a Head-MountedDisplay (HMD) together with an EEG headset can prove to be difficult given that the EEG sensors must stay on position. Choosing an EEG headset becomes a complex task if we consider mobility and comfort requirements. Equipment presented in Fig. 1 and Fig. 2 must be redesigned to be used in real situations. Some of equipment use wires or cables, and most of them require application of gel or saline substance to capture mental signals with high precision. The contribution of $\mathrm{HCI}$ in this BCI issue is exactly proposing the redesign of such equipment considering, for example, accessibility, usability, and ergonomic aspects. An ideal BCI headset must not use wires or cables, which hamper mobility; neither use gel or saline solutions, which are one more component to be carried on and make headset's use more difficult. We understand that this device must be lightweight and without additional parts, for example, batteries.

In BCIs, system needs to constantly adapt itself to user's signals. This adjustment must be fast and with precision. Current BCIs present a very low information transmission speed rate, being necessary, for example, almost two minutes to "digitalize" a simple word. Nowadays, this challenge is minimized with the use of word completers, accelerating the speed, as described in [15]. The BCI precision does not always reach a satisfactory value, mainly in BCI based on visual stimulus. Sometimes, actions repetition or undo are required, causing discomfort or even discontent in the usage of interactive systems with this kind of interface. The sum of these factors may generate frustrations to user and, consequently, resistance to the BCI usage. Furthermore, the performance of low-cost BCI commercial devices must be investigated, such as EPOC which presents less electrodes than other EEG headsets with medical purposes. In a comparison performed by Al-Zubi et al. [1], this headset with 14 electrodes presented only $5 \%$ fewer precision than a professional EEG headset with 128 electrodes. 
BCI tests and experiments are often conducted in controlled environments, in laboratory, that does not correspond to the real context of use of desktop computers, where users usually perform different tasks in parallel and work simultaneously, breaking their concentration constantly, either to answer the phone or to fetch a glass of water. This fact seems to demonstrate that currently asynchronous BCIs have more advantages in real situations, since they provide greater facility to the user when performing tasks in parallel, without prejudicing interaction with computer.

BCIs with wireless headsets are more practical and comfortable. Many manufacturers state that EEG signals are encrypted before transferred to device. However, it is necessary to note that, in a future with massive use of BCIs, cryptography breaking enables attackers to capture cerebral waves, which transmit not only commands but also mental states and feelings. As BCI area progresses, this challenge has more severe consequences since the higher the precision and greater the amount of information, the greater is the risk of privacy loss. Looking forward, when BCI technologies reach advanced stage, information espionage will no longer use phone tapping and network sniffers. Instead, it will use mind tapping and cerebral signals sniffers. Thus, with research advancements, new challenges arise and the presented survey is taken as starting point to development and evolution of BCIs.

\section{DISCUSSION}

The brain is in constant activity and humans think all the time, even while they are asleep or dreaming. When a person uses a computer, an information wave is lost, e.g. concentration level, frustrations, cognitive workload and user's tension. This information could be used in BCIs, enabling interactive systems to adjust to their users, for example, changing the amount of text and figures [25], changing desktop screen sizes to control resources [30] and to offer more space to important applications, identifying and correcting interaction mistakes [32], choosing a more suitable video to user [36], or even presenting more relevant information based on the user cerebral activity.

Invasive BCIs based on implanted chips using biocompatible materials are mature enough to enable monkeys to control a mechanical arm only with thought, as for example presented by Carmena et al. [3]. However, it requires a long period of training. Also, the invasion levels, costs and health risks make it an unviable procedure to healthy humans. Whereas noninvasive BCIs are not mature enough to be used as a single input, although it may already be adopted on multimodal interfaces efficiently in real usage scenarios. In addition, BCIs without stimulus, in which a simple thought would control an entire system, are the most desired ones. However, with current technologies, without stimulus, it is only possible to recognize mental states with certain precision, such as concentration.

Thus, based on the BCI literature review, we identified that most of noninvasive BCIs depends on visual stimulus to reach satisfactory precision and speed (see Table I). Such a care with design becomes even more important in these interfaces, since input/output depends on system's graphical interface. BCIs based on sound stimulus also face a similar difficult, because these BCIs require a special concern regarding warning sounds in order to sounds do not disturb the feedback to sound stimulus.

There are no ready-to-use models in which we can model interaction between users and computers via brain waves. It is henceforth necessary to develop methods, techniques, approaches and technologies to greater support works in this area. The interaction documentation of a specific user based on information captured from user's brain may be used to evaluate interfaces and indicate, as a result, the system's intuitiveness. Moreover, user's behavior pattern directly captured from brain activity may contribute to enhance the interaction quality of adaptive interfaces since system may learn about user's behavior and automatically provide a molded interface.

For widespread use of this interaction form, it is necessary to face current limitations and overcome its challenges. Whereas precision involves partially HCI area, concentration, speed, comfort, environment, difficulty in its usage, and privacy are constant concerns of interactive systems. The fact that most of works using noninvasive BCIs needs visual stimulus increases the need of a greater concern from HCI and VR communities in BCI research. Even in BCIs without visual stimulus, these areas may contribute since it is important to consider the feedback, making the command more intuitive with HCI and more immersive with VR. BCI technologies are a fundamental step to more transparent ubiquous interactions, in which we'll control different devices simply by our "will". Through capture and identification our thoughts, no effort will be required in daily interactions with devices. Using muscles will not be necessary, except those ones responsible for vital activities of organism, such as involuntary movements of heartbeat.

We believe that, if HCI knowledge is associated from the beginning, it is possible to advance toward more comfortable, suitable and easy-to-use BCIs, so that users may have a higher degree of satisfaction in interactive systems. Our research group is exploring this area, in order to purpose new BCIs guided by human factors, which are involved in this high complex form of (brain)human-computer interaction. In the same way, we believe that the area of VR plays a vital role in brain-computer interactions, providing an immersive environment, easing movement imagination and increasing focus on visual stimulus.

\section{CONCLUSION}

This paper presented a survey of BCI and additionally, based on this review, we identified and discussed several challenges for BCI in the interactive systems context. We believe that these challenges must be addressed so that BCIs may be adopted in interactive systems more effectively. Furthermore, due to advancements and price reduction of headsets, BCIs will be common in the near future as well as other kinds of interface/interaction are today, for example, such as those provided by mobile devices and by Kinect.

We are aware that thinking about interaction design in this domain involves various areas of knowledge, especially to 
reach its full potential. However, it is important to point out that HCI can contribute to the expansion of knowledge frontiers; outcomes achieved with this study is a concrete example since it enhances the importance of this review and outstands the merit of literature works. Considering different related areas and the diverse use possibilities of BCI, this research topic deserves greater attention from both $\mathrm{HCI}$ and VR communities in order to undertake further studies on interaction in BCIs.

As future works we make an interaction design study and we will implement a visual stimulus-based BCI game for use with a low-cost noninvasive EEG headset.

\section{ACKNOWLEDGMENT}

This work was partially supported by the Brazilian Federal Agency for Support and Evaluation of Graduate Education (CAPES) and by the Physical Artifacts of Interaction Research Group (PAIRG) at the Federal University of Rio Grande do Norte (UFRN), Brazil. The present paper is a extended and reviewed version of a previous work published at XI Simpósio Brasileiro sobre Fatores Humanos em Sistemas Computacionais (IHC'12) entitled "Interfaces CérebroComputador de Sistemas Interativos: Estado da Arte e Desafios de IHC" [38]. The authors thank JIS editors for the invitation.

\section{REFERENCES}

[1] H.S. Al-Zubi, N.S. Al-Zubi, and W. Al-Nuaimy, "Toward inexpensive and practical brain computer interface," in Proceedings of the Developments in E-systems Engineering (DeSE'11), IEEE, 2011, pp. 98-101, doi: 10.1109/DeSE.2011.116.

[2] A. Campbell, T. Choudhury, S. Hu, H. Lu, M.K. Mukerjee, M. Rabbi, and R.D.S. Raizada, "NeuroPhone: brain-mobile phone interface using a wireless EEG headset," in Proceedings of the $2^{\text {nd }}$ ACM SIGCOMM Workshop on Networking, Systems, and Applications on Mobile Handhelds (MobiHeld'10), ACM, 2010, pp. 3-8, doi: $10.1145 / 1851322.1851326$

[3] J.M. Carmena, M.A. Lebedev, R.E. Crist, J.E. O’Doherty, D.M. Santucci, D.F. Dimitrov, P.G. Patil, C.S. Henriquez, and M.A.L. Nicolelis, "Learning to control a brain-machine interface for reaching and grasping by primates," in PLoS Biology, vol. 1, n. 2, 2003, pp. 193208, doi: 10.1371/journal.pbio.0000042.

[4] H. Cecotti, "A self-paced and calibration-less SSVEP-based braincomputer interface speller," in IEEE Transactions on Neural Systems and Rehabilitation Engineering, vol. 18, n. 2, IEEE, 2010, pp. 127-133, doi: 10.1109/TNSRE.2009.2039594.

[5] P. Coulton, C.G. Wylie, and W. Bamford, "Brain interaction for mobile games," in Proceedings of the $15^{\text {th }}$ International Academic MindTrek Conference (MindTrek'11), ACM, 2011, pp. 37-44, doi: $10.1145 / 2181037.2181045$

[6] P. Gergondet, S. Druon, A. Kheddar, C. Hintermuller, C. Guger, and M. Slater, "Using brain-computer interface to steer a humanoid robot," in Proceedings of the IEEE International Conference on Robotics and Biomimetics (ROBIO'11), IEEE, 2011, pp. 192-197, doi: 10.1109/ROBIO.2011.6181284.

[7] M. Grierson and C. Kiefer, "Better brain interfacing for the masses: progress in event-related potential detection using commercial brain computer interfaces," in Proceedings of the Extended Abstracts on Human Factors in Computing Systems (CHI EA'11), ACM, 2011, pp. 1681-1686, doi: 10.1145/1979742.1979828.

[8] C. Guger, G. Krausz, B.Z. Allison, and G. Edlinger, "Comparison of dry and gel based electrodes for P300 brain-computer interfaces," in Frontiers in Neuroprosthetics, vol. 6, Frontiers, 2012, pp. 1-7, doi: 10.3389/fnins.2012.00060.
[9] G. Hakvoort, H. Gürkök, D.P-O. Bos, M. Obbink, and M. Poel, "Measuring immersion and affect in a brain-computer interface game," in Proceedings of the $13^{\text {th }}$ IFIP TC 13 International Conference on Human-Computer Interaction (INTERACT'11), Springer, 2011, pp. 115-128, doi: 10.1007/978-3-642-23774-4_12.

[10] C. Harrison, D. Tan, and D. Morris, "Skinput: appropriating the body as an input surface," in Proceedings of the ACM CHI Conference on Human Factors in Computing Systems (CHI'10), ACM, 2010, pp. 453462, doi: $10.1145 / 1753326.1753394$.

[11] B.A.S. Hasan and J.Q. Gan, "Hangman BCI: an unsupervised adaptive self-paced brain-computer interface for playing games," in Computers in Biology and Medicine, vol. 42, n. 5, Elsevier, 2012, pp. 598-606, doi: 10.1016/j.compbiomed.2012.02.004.

[12] N.J. Hill and B. Schölkopf, "An online brain-computer interface based on shifting attention to concurrent streams of auditory stimuli," in Journal of Neural Engineering, vol. 9, n. 2, pp. 1-13, 2012, doi: 10.1088/1741-2560/9/2/026011.

[13] H-J. Hwang, J-H. Lim, Y-J. Jung, H. Choi, S.W. Lee, and C-H. Im, "Development of an SSVEP-based BCI spelling system adopting a QWERTY-style LED keyboard," in Journal of Neuroscience Methods, vol. 208, n. 1, Elsevier, 2012, pp. 59-65, doi: 10.1016/j.jneumeth.2012.04.011.

[14] C. Kapeller, C. Hintermüller, and C. Guger, "Usability of videooverlaying SSVEP based BCIs," in Proceedings of the $3^{\text {rd }}$ Augmented Human International Conference (AH'12), ACM, 2012, doi: 10.1145/2160125.2160151.

[15] T. Kaufmann, S. Völker, L. Gunesch, and A. Kübler, "Spelling is just a click away - a user-centered brain-computer interface including autocalibration and predictive text entry," in Frontiers in Neuroprosthetics, vol. 6, Frontiers, 2012, pp. 1-10, doi: 10.3389/fnins.2012.00072.

[16] D-W Kim, J-H Cho, H-J Hwang, J-H Lim, and C-H Im, “A vision-free brain-computer interface (BCI) paradigm based on auditory selective attention," in Proceedings of the Annual International Conference of the IEEE Engineering in Medicine and Biology Society (EMBC'11), IEEE, 2011, pp. 3684-3687, doi: 10.1109/IEMBS.2011.6090623.

[17] S.S. Liu, A. Rawicz, S. Rezaei, T. Ma, C. Zhang, K. Lin, and E. Wu, "An eye-gaze tracking and human computer interface system for people with ALS and other locked-in diseases," in Journal of Medical and Biological Engineering, vol. 32, n. 2, pp. 37-42, 2012.

[18] T. Liu, L. Goldberg, S. Gao, and B. Hong, "An online brain-computer interface using non-flashing visual evoked potentials," in Journal of Neural Engineering, vol. 7, n. 3, 2010, pp. 1-9, doi: 10.1088/17412560/7/3/036003.

[19] F. Lotte, J. Fujisawa, H. Touyama, R. Ito, M. Hirose, and A. Lécuyer, "Towards ambulatory brain-computer interfaces: a pilot study with P300 signals," in Proceedings of the International Conference on Advances in Computer Enterntainment Technology (ACE'09), ACM, 2009, pp. 336339, doi: 10.1145/1690388.1690452.

[20] J. Mankoff, A. Dey, U. Batra, and M. Moore, "Web accessibility for low bandwidth input," in Proceedings of the $5^{\text {th }}$ ACM International Conference on Assistive Technologies (ASSETS'02), ACM, 2002, pp. 17-24, doi: 10.1145/638249.638255.

[21] M. Mauro, P. Francesco, S. Stefano, G. Luciano, and P. Konstantinos, "Spatial attention orienting to improve the efficacy of a brain-computer interface for communication," in Proceedings of the $9^{\text {th }}$ ACM SIGCHI Italian Chapter International Conference on Computer-Human Interaction (CHItaly'11), ACM, 2011, pp. 114-117, doi: 10.1145/2037296.2037325.

[22] J.D.R. Millán, R. Rupp, G.R. Müller-Putz, R. Murray-Smith, C. Giugliemma, M. Tangermann, C. Vidaurre, F. Cincotti, A. Kübler, R. Leeb, C. Neuper, K.R. Müller, and D. Mattia, "Combining braincomputer interfaces and assistive technologies: state-of-the-art and challenges," in Frontiers in Neuroprosthetics, vol. 4, Frontiers, 2010, pp. 1-33, doi: 10.3389/fnins.2010.00161.

[23] E.M. Mugler, C.A. Ruf, S. Halder, M. Bensch, and A. Kubler, "Design and implementation of a P300-based brain-computer interface for controlling an internet browser," in IEEE Transactions on Neural Systems and Rehabilitation Engineering, vol. 18, n. 6, IEEE, 2010, pp. 599-609, doi: 10.1109/TNSRE.2010.2068059. 
[24] Y. Nam, Q. Zhao, A. Cichocki, and S. Choi, "Tongue-Rudder: a glossokinetic-potential-based tongue-machine interface," in IEEE Transactions on Biomedical Engineering, vol. 59, n. 1, IEEE, 2012, pp. 290-299, doi: 10.1109/TBME.2011.2174058.

[25] A. Nijholt, D. Tan, B. Allison, J.R. Milan, and B. Graimann, "Braincomputer interfaces for $\mathrm{HCI}$ and games," in Proceedings of the Extended Abstracts on Human Factors in Computing Systems (CHI EA'08), ACM, 2008, pp. 3925-3928, doi: 10.1145/1358628.1358958.

[26] G. Pires, U. Nunesa, and M. Castelo-Branco, "Statistical spatial filtering for a P300-based BCI: tests in able-bodied, and patients with cerebral palsy and amyotrophic lateral sclerosis," in Journal of Neuroscience Methods, vol. 195, n. 2, Elsevier, 2011, pp. 270-281, doi: 10.1016/j.jneumeth.2010.11.016.

[27] G.M. Poor, L.M. Leventhal, S. Kelley, J. Ringenberg, and S.D. Jaffee, "Thought cubes: exploring the use of an inexpensive brain-computer interface on a mental rotation task," in Proceedings of the $13^{\text {th }}$ International ACM SIGACCESS Conference on Computers and Accessibility (ASSETS'11), ACM, 2011, pp. 291-292, doi: $10.1145 / 2049536.2049612$.

[28] A.B. Randolph, "Not all created equal: individual-technology fit of brain-computer interfaces," in Proceedings of the $45^{\text {th }}$ Hawaii International Conference on System Science (HICSS'12), IEEE, 2012, pp. 572-578, doi: 10.1109/HICSS.2012.451.

[29] E.T. Solovey, A. Girouard, K. Chauncey, L.M. Hirshfield, A. Sassaroli, F. Zheng, S. Fantini, and R.J.K. Jacob, "Using fNIRS brain sensing in realistic HCI settings: experiments and guidelines," in Proceedings of the $22^{\text {nd }}$ Annual ACM Symposium on User Interface Software and Technology (UIST'09), ACM, 2009, pp. 157-166, doi: $10.1145 / 1622176.1622207$

[30] E. Solovey, P. Schermerhorn, M. Scheutz, A. Sassaroli, S. Fantini, and R. Jacob, "Brainput: enhancing interactive systems with streaming fNIRS brain input," in Proceedings of the ACM CHI Conference on Human Factors in Computing Systems (CHI'12), ACM, 2012, pp. 21932202, doi: $10.1145 / 2207676.2208372$.

[31] S. Vernon and S.S. Joshi, "Brain-muscle-computer interface: mobilephone prototype development and testing," in IEEE Transactions on Information Technology in Biomedicine, vol. 15, n. 4, IEEE, 2011, pp. 531-538, doi: 10.1109/TITB.2011.2153208.

[32] C. Vi and S. Subramanian, "Detecting error-related negativity for interaction design," in Proceedings of the ACM CHI Conference on Human Factors in Computing Systems (CHI'12), ACM, 2012, pp. 493502, doi: 10.1145/2207676.2207744.

[33] Y-T. Wang, Y. Wang, and T-P. Jung, "A cell-phone-based braincomputer interface for communication in daily life," in Journal of Neural Engineering, vol. 8, n. 2, 2011, doi: 10.1088/17412560/8/2/025018.

[34] J.R. Wolpaw, N. Birbaumer, D.J. McFarland, G. Pfurtscheller, and T.M. Vaughan, "Brain-computer interfaces for communication and control," in Clinical Neurophysiology, vol. 113, n. 6, Elsevier, 2002, pp. 767-791, doi: 10.1016/S1388-2457(02)00057-3.

[35] H. Xu, T. Qian, B. Hong, X. Gao, and S. Gao, "Brain-actuated human computer interface for google search," in Proceedings of the $2^{\text {nd }}$ International Conference on Biomedical Engineering and Informatics (BMEI'09), IEEE, 2009, pp. 1-4, doi: 10.1109/BMEI.2009.5305708.

[36] A. Yazdani, J-S. Lee, J-M. Vesin, and T. Ebrahimi, "Affect recognition based on physiological changes during the watching of music videos," in ACM Transactions on Interactive Intelligent Systems, vol. 2, n. 1, ACM, 2012, pp. 1-26, doi: 10.1145/2133366.2133373.

[37] B.F. Yuksel, M. Donnerer, J. Tompkin, and A. Steed, "A novel braincomputer interface using a multi-touch surface," in Proceedings of the
ACM CHI Conference on Human Factors in Computing Systems (CHI'10), ACM, 2010, pp. 855-858, doi: 10.1145/1753326.1753452.

[38] A.L.S. Ferreira, L.C. Miranda, and E.E.C. Miranda, "Interfaces cérebrocomputador de sistemas interativos: estado da arte e desafios de IHC," in Anais do XI Simpósio Brasileiro sobre Fatores Humanos em Sistemas Computacionais (IHC'12), SBC, 2012, pp. 239-248.

[39] R. Leeb, M. Lancelle, V. Kaiser, D. Fellner, and G. Pfurtscheller, "Thinking Penguin: multimodal brain-computer interface control of a VR game," in IEEE Transactions on Computational Intelligence and AI in Games, vol. 5, n. 2, IEEE, 2013, pp. 117-128, doi: 10.1109/TCIAIG.2013.2242072.

[40] M. Marchesi, "From mobie to Neu: 3D animated contents controlled by a brain-computer interface," in Proceedings of the Virtual Reality International Conference (VRIC'12), ACM, 2012, pp. 1-3, doi: 10.1145/2331714.2331747.

[41] R. Poli, C. Cinel, A. Matran-Fernandez, F. Sepulveda, and A. Stoica, "Towards cooperative brain-computer interfaces for space navigation," in Proceedings of the International Conference on Intelligent User Interfaces (IUI'13), ACM, 2013, pp. 149-160, doi: 10.1145/2449396.2449417.

[42] D. Hood, D. Joseph, A. Rakotonirainy, S. Sridharan, and C. Fookes, "Use of brain computer interface to drive: preliminary results," in Proceedings of the $4^{\text {th }}$ International Conference on Automotive User Interfaces and Interactive Vehicular Applications (AutomotiveUI'12), ACM, 2012, pp. 103-106, doi: 10.1145/2390256.2390272.

[43] G. Edlinger, C. Holzner, and C. Guger, "A hybrid brain-computer interface for smart home control," in Proceedings of the $14^{\text {th }}$ International Conference on Human-Computer Interaction (HCII'11), Springer, 2011, pp. 417-425, doi: 10.1007/978-3-642-21605-3_46.

[44] G. Edlinger and C. Guger, "A hybrid brain-computer interface for improving the usability of a smart home control," in Proceedings of the ICME International Conference on Complex Medical Engineering (CME'12), IEEE, 2012, pp. 182-185, doi: 10.1109/ICCME.2012.6275714.

[45] C. Escolano, J.M. Antelis, and J. Minguez, "A telepresence mobile robot controlled with a noninvasive brain-computer interface," in IEEE Transactions on Systems, Man, and Cybernetics, Part B: Cybernetics, vol. 42, n. 3, IEEE, 2012, pp. 793-804, doi: 10.1109/TSMCB.2011.2177968.

[46] S.M. Grigorescu, T. Lüth, C. Fragkopoulos, M. Cyriacks, and A. Gräser, "A BCI-controlled robotic assistant for quadriplegic people in domestic and professional life," in Robotica, vol. 30, n. 3, 2012, pp. 419-431, doi: 10.1017/S0263574711000737.

[47] G. Pfurtscheller, R. Leeb, C. Keinrath, D. Friedman, C. Neuper, C. Guger, and M. Slater, "Walking from thought," in Brain Research, vol. 1071, 2006, pp. 145-152.

[48] R. Leeb, D. Friedman, G.R. Müller-Putz, R. Scherer, M. Slater, and G. Pfurtscheller, "Self-paced (asynchronous) BCI control of a wheelchair in virtual environments: a case study with a tetraplegic," in Computational Intelligence and Neuroscience, vol. 2007, 2007, pp. 1-8, doi: $10.1155 / 2007 / 79642$.

[49] D. Friedman, R. Leeb, G. Pfurtscheller, and M. Slater, "Humancomputer interface issues in controlling virtual reality with braincomputer interface," in Human-Computer Interaction, vol. 25, n. 1, Taylor \& Francis, 2010, pp. 67-94, doi: 10.1080/07370020903586688.

[50] Lotte, Fabien, Josef Faller, Christoph Guger, Yann Renard, Gert Pfurtscheller, Anatole Lécuyer, and Robert Leeb. "Combining BCI with virtual reality: towards new applications and improved BCI," Towards Practical Brain-Computer Interfaces, pp. 197-220. Springer, 2013, doi: 10.1007/978-3-642-29746-5_10. 